\title{
Experimental Demonstration of Generalised Space Shift Keying for Visible Light Communication
}

\author{
Rui Bian ${ }^{1}$, Stefan Videv ${ }^{1}$, Alexander D. Griffiths ${ }^{2}$, Jonathan J. D. McKendry ${ }^{2}$, Enyuan Xie ${ }^{2}$, Erdan Gu$^{2}$, Martin \\ D. Dawson ${ }^{2}$, and Harald Haas ${ }^{1}$ \\ ${ }^{1}$ University of Edinburgh, Email:\{r.bian, s.videv, h.haas\}@ed.ac.uk \\ ${ }^{2}$ University of Strathclyde, Email:\{alex.griffiths, jonathan.mckendry, enyuan.xie, erdan.gu, \\ m.dawson\}@strath.ac.uk
}

\begin{abstract}
A low complexity generalised space shift keying (GSSK) experimental set-up for visible light communication (VLC) is demonstrated. The GSSK encoder is implemented in a field programmable gate array (FPGA) board. No digitalto-analog converter (DAC) is required and up to 16 output channels are supported which greatly exceeds that of an arbitrary waveform generator (AWG). A $4 \times 4$ Gallium Nitride (GaN) micro-LED array is used as transmitter while 4 avalanche photo diode (APD) receiver boards are acting as receivers. GSSK exploits the natural differences between the multiple communication links. The bit error ratio (BER) performances are evaluated for different transmitter and receiver arrangements. It is also shown that how different receiver positions and increasing receiver number will affect the BER performance. The bit error performance greatly depends on the dissimilarity of the channel gains. A spectral efficiency of $\mathbf{1 6}$ bits/symbol is achieved by using all 16 micro-LEDs and 4 receivers. The implementation of the experiment is introduced in detail and experimental results are given.
\end{abstract}

Index Terms-Visible light communication, optical modulation, space shift keying, spatial modulation, micro-LED, FPGA.

\section{INTRODUCTION}

$\mathbf{L}$ IGHT emitting diodes (LEDs) are widely used for illumination and in recent years they have been given a new use - transmitting high speed wireless data. This is referred to as visible light communication (VLC). VLC has now become a rapidly advancing technology for optical wireless communication (OWC). VLC complements Radio Frequency (RF) technology and offers advantages in terms of capacity, efficiency, safety and security.

In VLC, visible light is the carrier of information and it is restricted to intensity modulation (IM) and direct detection (DD). Incoherent solid-state lighting elements such as LEDs are widely employed as VLC transmitters. The off-the-shelf commercial LEDs are designed for lighting and their $-3 \mathrm{~dB}$ bandwidth are typically $2-3 \mathrm{MHz}$ [1]. To achieve a higher communication data rate, great effort is directed towards developing higher bandwidth LEDs. The work in [2] introduced a Gallium Nitride (GaN) LED with diameter of $50 \mu \mathrm{m}$. With one single such micro-LED, an optical link with data rates up to $3 \mathrm{Gbits} / \mathrm{s}$ was demonstrated in [3]. As a trade-off, such high speed link requires a highly complex digital modulation scheme.
A wide range of modulation techniques have been proposed for VLC. Turning the LED on and off to represent binary information is known as on-off-keying (OOK). It offers low spectral efficiency but benefits from low complexity in implementation and is robust against LED non-linearity. Pulse amplitude modulation (PAM) and optical orthogonal frequency division multiplexing (OFDM) are also studied and compared in [4], [5] and [6]. Optical-OFDM offers high spectral efficiency but suffers from the LED non-linearity [7]. Solutions including predistortion are presented to counter the effect of LED nonlinearity, but these measures require additional computational complexity. Multiple input multiple output (MIMO) techniques which offer high data rates by increasing the spectral efficiency are also studied for VLC [8]. In a typical MIMO system, all the transmitters are active during transmission and this causes inter-channel interference which should be mitigated at the cost of additional computational complexity.

Spatial modulation (SM), introduced in [9] and further enhanced in [10] and [11], describes a new low-complexity implementation for MIMO wireless systems. In SM, some information bits are conveyed in the additional spatial dimension which is the physical position of each transmitter. During each symbol transmission, only one transmitter is active so that inter-channel interference is avoided. In addition, the number of receivers can be smaller than the number of transmitters. SM is also studied for VLC in $[12,13]$ and is combined with OFDM to achieve high spectral efficiency. Space Shift Keying (SSK) [14] and generalised SSK (GSSK) [15] are special cases of SM where only spatial information is sent and each individual transmitter operates in OOK mode. SSK and GSSK have all been expanded to optical communication $[16,17]$. Compared to OOK, SSK and GSSK inherit the robustness against LED non-linearity, but offer higher spectral efficiency by exploiting the spatial dimension for information transmission. Compared to PAM and direct current biased optical OFDM (DCO-OFDM), SSK and GSSK require much lower complexity in implementation and digital-to-analog converters (DACs) are not required which significantly reduce the complexity and the cost.

Both SSK and GSSK are well studied for optical communication [16-18] but there are little experimental results. Work in [19] and [20] present both SSK and GSSK using the digital camera as receiver. Image sensor receivers have 
several advantages against photo diodes (PDs) as the LED sources are well separated on the image because there are a large number of pixels which can detect the LED lights. Thus a higher order operation can be achieved easily by using more LEDs and the lights are prevented from being mixed by a high resolution camera receiver. However the data rate is limited by the camera's frame rate - up to 1000 frames per second as reported. The work in [21] shows an experimental set-up with 2 LEDs and 1 PD receiver and states that the experimental modulation order is limited by the Arbitrary Waveform Generator (AWG) which contains only two output ports.

This work presents a low-complexity GSSK experimental set-up with a FPGA based GSSK encoder while employing a 4 by 4 micro-LED array as transmitter and 4 avalanche photo-diode (APD) receiver boards. Up to 16 micro-LEDs can be activated for a high order GSSK operation. The bit error performances for differnet experimental set-up are evaluated.

The remainder of this paper is organised as follows: In Section II the concept of GSSK is introduced. In Section III the system set-up in detail is discussed. Results and discussions are given in Section IV. Finally, conclusions are presented in Section V.

\section{Generalised Space Shift Keying}

A SSK system consists of a transmitter array and a receiver array. Considering a LED array with $N_{t}$ LEDs, each LED has a unique spatial position and it can be represented by a unique binary index. In SSK operation, only one of the LEDs is active during each symbol duration. A maximum likelihood (ML) decoder is implemented on the receiver end to determine which LED was turned on based on the natural differences of channel gains between each LED and the receivers. Once the activated LED is found, its binary index is decoded as the transmitted data which is in $\log _{2}\left(N_{t}\right)$ bits. Thus a SSK operation with $N_{t}$ LEDs offers a spectral efficiency of $\log _{2}\left(N_{t}\right)$ bits per symbol.

This operation can be generalised using GSSK by removing the limitation of turning on only one LED during each symbol duration. For a LED array of $N_{t}$ LEDs, there are $2^{N_{t}}$ combinations of modulating LEDs including turning off all the LEDs. Thus in the $N_{t}$ GSSK system the maximum spectral efficiency is $N_{t}$ bits per symbol.

During a symbol clock $T$, a duty cycle $\tau$ can be introduced to control how long the LED will be on during each symbol duration. A non-return-to-zero (NRZ) pulse pattern is formed when $\tau=1$ and each LED is on for the whole symbol duration. Using a NRZ pulse pattern offers the maximum spectral efficiency of $N_{t}$ bits per symbol. When $\tau<1$ each LED is turned on for part of each symbol duration then turned off and the signal is in the return-to-zero (RZ) pulse pattern. The RZ pattern has better performance against signal's baseline drifting and it is helpful for synchronisation purposes. Varying the duty cycle will also make the LEDs work in dimmed conditions which supports the IEEE 802.15.7 standard [22]. An example GSSK scheme for two LEDs when $\tau=1$ is shown in Fig. 1.

\begin{tabular}{|c|c|c|c|}
\hline Symbol & Binary & LED1 & LED2 \\
\hline $\mathbf{0}$ & {$[00]$} & OFF & OFF \\
\hline $\mathbf{1}$ & {$\left[\begin{array}{l}0 \\
1\end{array}\right]$} & OFF & ON \\
\hline $\mathbf{2}$ & {$[10]$} & ON & OFF \\
\hline $\mathbf{3}$ & {$\left[\begin{array}{ll}1 & 1\end{array}\right]$} & ON & ON \\
\hline
\end{tabular}

Fig. 1: Example of GSSK scheme for two LEDs

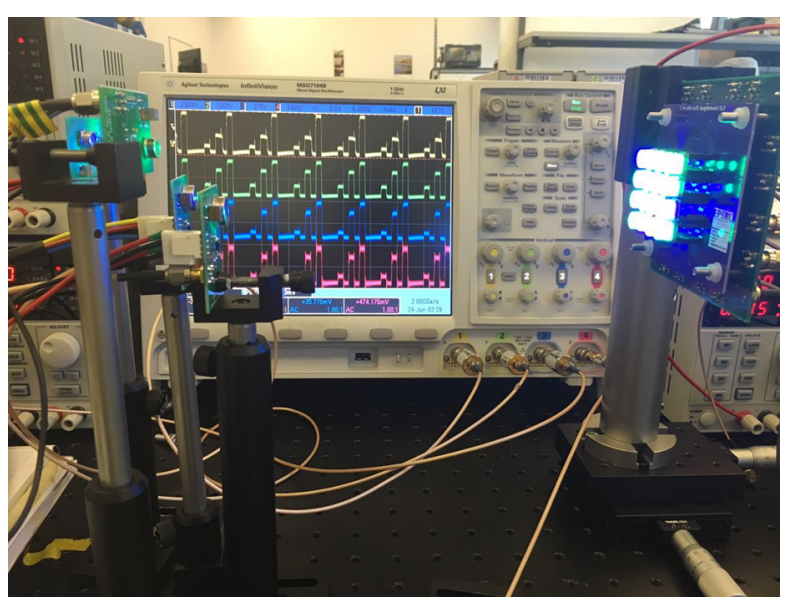

Fig. 2: Experimental Set-up

TABLE I

\begin{tabular}{c|c}
\hline Receiver Position & Receiver coordinate $(\mathrm{cm})$ \\
\hline 1 & $(35,0.5,20.5)$ \\
2 & $(35,2.5,20.5)$ \\
3 & $(37,0.5,22.5)$ \\
4 & $(37,0.5,22.5)$ \\
5 & $(35,0.5,21.5)$ \\
\hline
\end{tabular}

\section{The EXPERIMENTAL SET-UP AND DESCRIPTION}

The experimental set-up is shown in Fig. 2. At the transmitter side, a GSSK encoder is implemented with a Nexys3 board, which features the Xilinx Spartan-6 LX16 field programmable gate array (FPGA). The 16 micro-LEDs are plugged into a daughterboard with $1 \mathrm{~cm}$ distance between each forming a 4 by 4 array. The daughterboard is attached to a motherboard which connects to DC power supply and 16 individual output ports of the FPGA. Each micro-LED has a plastic aspheric lens to collimate the light and this reduces the semi-angle to 4 degrees. The 16 micro-LEDs are numbered from 1 to 16 starting from the top left corner one to the lower right corner one. At the receiver end, up to 4 individual APD receiver boards are available and no lens is used on the receiver side in this work. The receivers are set to different positions regarding different experiments and the coordinates of these positions 


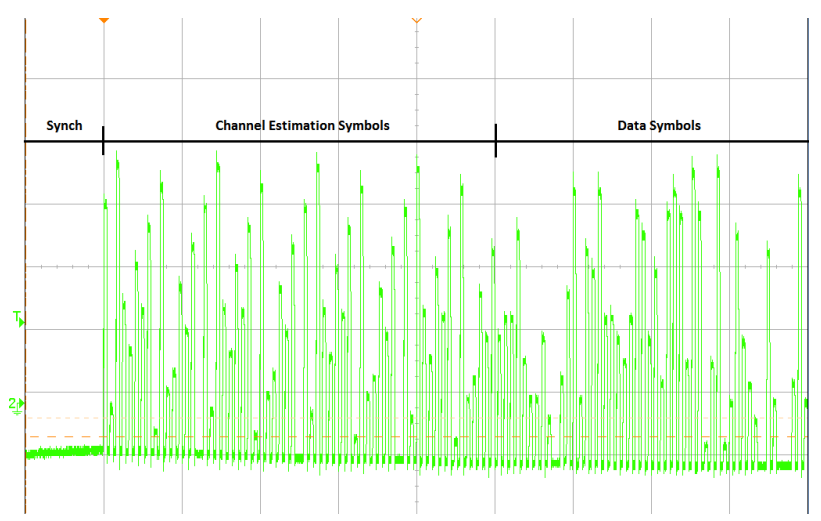

Fig. 3: Captured signal when $\tau=0.5$

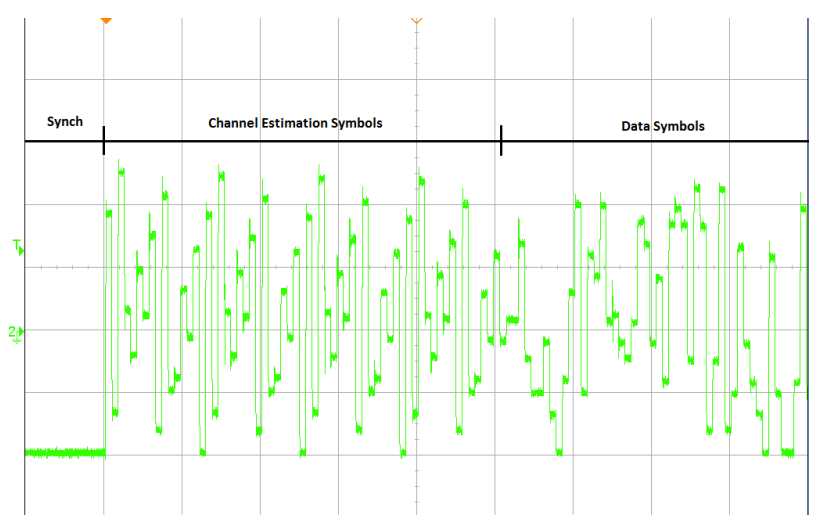

Fig. 4: Captured signal when $\tau=1$

are given in Table I. The link distance is $35 \mathrm{~cm}$ or $37 \mathrm{~cm}$ depending on the receiver positions.

The data packets for measurements are generated by the FPGA and contain three parts: the synchronisation symbols, channel estimation symbols and data symbols. The synchronisation symbols are used to trigger the signal. In the channel estimation part, all the possible combinations of LEDs are activated sequentially and this entire sequence is sent repeatedly for 4 cycles. The purpose of this is to train the ML decoder by capturing the power level for each known GSSK symbol. After the estimation symbols, $6.4 \times 10^{4}$ bits of data are encoded and sent. In the designed FPGA encoder, varying the duty cycle $\tau$ is supported which shows that the encoder is a potential candidate for VLC application where dimmed control is required. Fig. 3 and Fig. 4 show the sample captured signals when $\tau=0.5$ and $\tau=1$ respectively.

There are four sets of measurements. The first experiment measures the channel gain between 4 selected LEDs and 2 receivers at different positions. The result shows how each channel gain differs from the others. The second is measuring the bit error ratio (BER) performance when using a single receiver and multiple transmitters. The third experiment shows how the BER performance behaves when setting receiver at different positions and using more receivers. The last experiment employs the entire 16 LEDs and 4 receivers measuring the BER performance against the achieved data rate while running at different spectral efficiencies. The switching frequency for each LED is set to $5 \mathrm{MHz}$ where $5 \mathrm{M}$ symbols

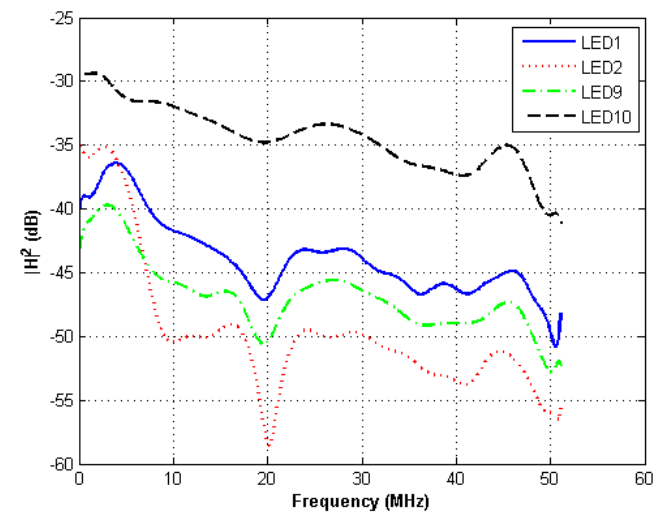

Fig. 5: Channel Gain: receiver position 1

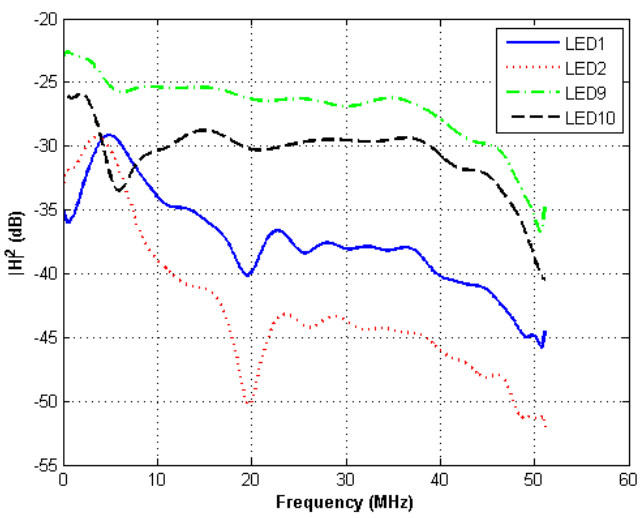

Fig. 6: Channel Gain: receiver position 2

are sent per second.

\section{RESULTS AND DisCUSSION}

The experiments evaluate the channel gains and BER performances at different spectral efficiencies. Four micro-LEDs are chosen from the array for the first three experiments. The LED numbers and their coordinates are given as: LED1 $(0,0$, 23), $\operatorname{LED} 2(0,1,23), \operatorname{LED} 9(0,0,21)$ and $\operatorname{LED} 10(0,1,21)$. As the direction of each LED is irregular when being plugged into the daughterboard, all the four LEDs are tilted towards position 5 resulting in forming an overlapping covering area.

In the first experiment, the APD receiver is set at position 1 and then the channel gains between each of the four LED and the receiver are measured. The result in Fig. 5 shows the natural differences of channel gains between each LED and the same receiver. These differences are exploited at the receiver to estimate which symbol has been transmitted. The measurement is then repeated by setting the receiver to position 2 and the result is shown in Fig. 6. By comparing the two figures it is clear that the channel gain also differs between links from one LED to receivers at different positions. This is a key feature exploited by GSSK. If receiver mobility is to be supported, the system needs to periodically send training information in order to to be able to decode information correctly. 


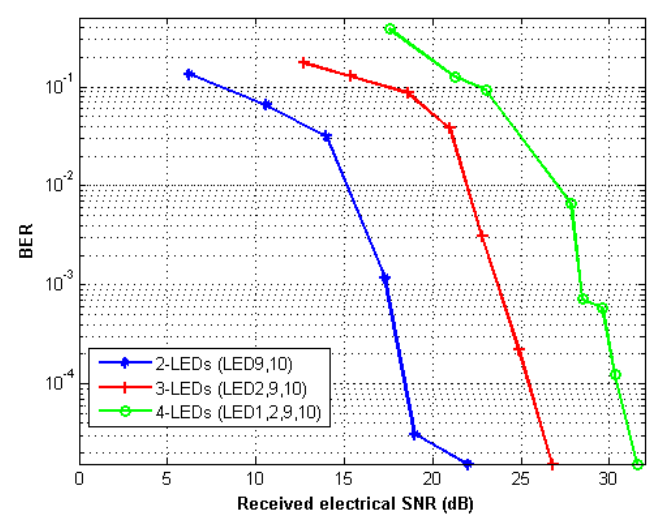

Fig. 7: BER vs received electrical SNR

In the second experiment, the error performance is evaluated when using one single receiver and multiple transmitters. Fig. 7 shows the BER performance against the received electrical signal-to-noise ratio (SNR) when using 2, 3 and 4 transmitters which results in a spectral efficiency of 2 bits/symbol, 3 bits/symbol and 4 bits/symbol respectively. The channel gain for LED1, LED2, LED9, LED10 are measured and then normalised giving $[0.14,0.244,0.477,1]$ respectively. The received signal for each case is similar to PAM signals at the same spectral efficiency. However the received power levels are not equally spaced. When the modulation order is increased, there are more GSSK symbols that need to be distinguished. The results show that when one more LED is used about $5 \mathrm{~dB}$ higher SNR is required for achieveing the similar BER of $1.56 \times 10^{-5}$ for this experimental set-up.

The error performance in the previous experiment is different when the receiver moves. Therefore, in the next experiment two receivers are put at position 1 and 2. The BER performance is firstly evaluated based on each receiver and then the received signals from both receivers are processed jointly. Results are shown in Fig. 8 and the measured normalised channel gain values for LED1, LED2, LED9 and LED10 are $[0.25,0.37,0.15,1]$ at position 1 and $[0.31,0.61,1,0.79]$ at position 2. As discussed in Fig. 5 and Fig. 6, the channel gain differs from the same LED set to the receiver at different positions. The result in Fig. 8 shows that the error performance of GSSK is strongly dependent on the dissimilarity of the channel gain values. In addition, by using two receivers the error performance is significantly improved.

In the last experiment all 16 micro-LEDs and 4 APD receivers are used. The measurements are taken while running GSSK at different spectral efficiencies up to 16 bits/symbol. Results are shown in Table II and the results are measured without applying forward error correction (FEC). Several switching frequencies for the LEDs are selected from $5 \mathrm{MHz}$ to $100 \mathrm{MHz}$. For a spectral efficiency of $8 \mathrm{bits} / \mathrm{symbol}$, the measured BER is $1.6 \times 10^{-5}$ at a switching frequency of $50 \mathrm{MHz}$ (i.e. $400 \mathrm{Mbits} / \mathrm{s}$ ). When the switching frequency is increased to $100 \mathrm{MHz}$ resulting in a data rate of $800 \mathrm{Mbits} / \mathrm{s}$, the BER goes up to $2 \times 10^{-2}$. This is because when the frequency increases, especially beyond the $-3 \mathrm{~dB}$ bandwidth

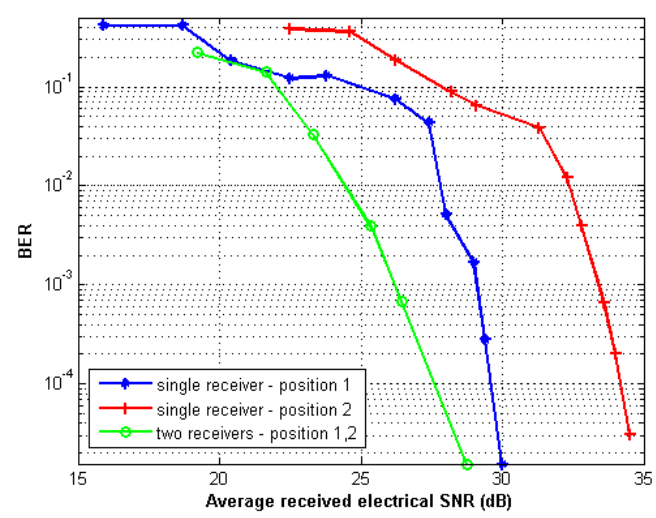

Fig. 8: BER vs received electrical SNR

of the LED, the power gain of each LED drops resulting in a lower received SNR. For a switching frequency of 50 $\mathrm{MHz}$ and a spectral efficiency of 12 bits/symbol, the BER is $1.6 \times 10^{-1}$ which is much higher than that of the case of 8 bits/symbol. Because as shown in previous result, it requires higher SNR for achieving the same BER performance at a higher spectral efficiency. The BER goes down while decreasing the switching frequency as the power gain of LED is higher at lower frequency. An operation at the maximum spectral efficiency of 16 bits/symbol while using 16 LEDs is demonstrated. The measured BER are $1.6 \times 10^{-5}$ and $1 \times 10^{-1}$ for $80 \mathrm{Mbits} / \mathrm{s}$ and $160 \mathrm{Mbits} / \mathrm{s}$. There are some restrictions for the current 16 by 4 experimental set-up. Currently only 4 receiver boards are used without using any lens and each of them has a small active area thus there is significant optical power loss. In some links only a small part of the optical power from the LED is captured by the receiver. For such links when the switching frequency increases, the channel SNR drops significantly thus errors occur. However, the demonstration still shows the high data rate potential of GSSK.

TABLE II

\begin{tabular}{|c|c|c|}
\hline $\begin{array}{c}\text { Spectral Efficiency } \\
\text { (bits/symbol) }\end{array}$ & $\begin{array}{c}\text { Data Rate } \\
\text { (Mbits/s) }\end{array}$ & BER \\
\hline 8 & 400 & $1.6 \mathrm{E}-5$ \\
\hline 8 & 800 & $2 \mathrm{E}-2$ \\
\hline 12 & 120 & $3.6 \mathrm{E}-4$ \\
\hline 12 & 300 & $8 \mathrm{E}-2$ \\
\hline 12 & 600 & $1.6 \mathrm{E}-1$ \\
\hline 16 & 80 & $1.6 \mathrm{E}-5$ \\
\hline 16 & 160 & $1 \mathrm{E}-1$ \\
\hline
\end{tabular}

\section{CONCLusions}

An experimental set-up of GSSK using a micro-LED array and APD receivers is demonstrated in this work. A FPGA based GSSK encoder is implemented which is capable of driving up to 16 LEDs. An experiment is carried out showing 
the natural differences between the channel gain values of different links. This is the key concept of GSSK. It is shown that the channel gains can be quite unique from LEDs to the same receiver or from one LED to different receivers. More experiments are carried out evaluating the BER performance with different set-up. The BER performance against received electrical SNR is presented in the cases of using 2, 3 and 4 LEDs with single receiver. For the demonstrated set-up, it is shown that about $5 \mathrm{~dB}$ higher $\mathrm{SNR}$ is required when one LED is added to the operation for achiecing the similar BER of $1.56 \times 10^{-5}$. Results also show that the BER performance varies when receivers change their positions. It is a limitation of GSSK that the error performance is highly dependent on the dissimilarity of the channel gain values. However the performance can be improved by applying more receivers which increases the data dimensions in the ML decoder. It is shown that about $4 \mathrm{~dB}$ and $8 \mathrm{~dB}$ lower SNR is required for achiecing the same BER of $10^{-3}$ while using two receivers compared to using single receiver at different positions respectively. The BER versus data rate measurements of applying different spectral efficiencies on a 16 by 4 GSSK set-up indicates the high data rate potential of GSSK. The BER results show that about $10^{-5}$ is achievable with the current setup for the spectral efficiencies from 8 bits/symbol up to 16 bits/symbol. For further study, the current experimental setup will be improved by employing more receivers and using lenses to reduce the optical power loss.

\section{ACKNOWLEDGMENT}

This research was supported by the UK Engineering and Physical Sciences Research Council under Grant $\mathrm{EP} / \mathrm{K} 00042 \mathrm{X} / 1$.

\section{REFERENCES}

[1] A. M. Khalid, G. Cossu, R. Corsini, P. Choudhury, and E. Ciaramella, "1-Gb/s transmission over a phosphorescent white LED by using rateadaptive discrete multitone modulation," IEEE Photon. J., vol. 4, no. 5, pp. 1465-1473, Oct. 2012.

[2] J. J. D. McKendry, D. Massoubre, S. Zhang, B. R. Rae, R. P. Green, E. Gu, R. K. Henderson, A. E. Kelly and M. D. Dawson, "Visiblelight communications using a CMOS-controlled micro-light-emittingdiode array," J. Lightw. Technol., vol. 30, no. 1, pp. 61-67, 2012.

[3] D. Tsonev, H. Chun, S. Rajbhandari, J. McKendry, S. Videv, E. Gu, M. Haji, S. Watson, A. Kelly, G. Faulkner, M. Dawson, H. Haas, and D. O'Brien, "A 3-Gb/s Single-LED OFDM-Based Wireless VLC Link Using a Gallium Nitride $\mu$ LED," IEEE Photon. Technol. Lett., vol. 26, no. 7, pp. 637-640, Apr. 2014

[4] K.-I. Ahn and J. Kwon, "Capacity analysis of m-pam inverse source coding in visible light communications," Journal of Lightwave Technology, vol. 30, no. 10, pp. 1399-1404, May 2012.

[5] J. Armstrong, "OFDM for Optical Communications," J. Lightw. Technol., vol. 27, no. 3, pp. 189-204, Feb. 2009.

[6] D. Barros, S. Wilson, and J. Kahn, "Comparison of orthogonal frequency-division multiplexing and pulse-amplitude modulation in indoor optical wireless links," IEEE Transactions on Communications, vol. 60, no. 1, pp. 153-163, 2012.

[7] H. Elgala, R. Mesleh, and H. Haas, "Impact of LED nonlinearities on optical wireless OFDM systems," in 2010 IEEE 21st International Symposium on Personal Indoor and Mobile Radio Communications (PIMRC), sept 2010, pp. $634-638$.
[8] T. Fath and H. Haas, "Performance Comparison of MIMO Techniques for Optical Wireless Communications in Indoor Environments," Communications, IEEE Transactions on, vol. 61, no. 2, pp. 733 - 742, 2013.

[9] R. Mesleh, H. Haas, C. W. Ahn, and S. Yun, "Spatial Modulation A New Low Complexity Spectral Efficiency Enhancing Technique," in IEEE International Conference on Communication and Networking in China (CHINACOM), Beijing, China, Oct. 25-27, 2006, pp. 1-5.

[10] R. Mesleh, H. Haas, S. Sinanović, C. W. Ahn, and S. Yun, "Spatial Modulation," IEEE Trans. on Veh. Tech., vol. 57, no. 4, pp. 2228 2241, Jul. 2008

[11] M. Di Renzo, H. Haas, and P. M. Grant, "Spatial modulation for multiple-antenna wireless systems: a survey," Communications Magazine, IEEE, vol. 49, no. 12, pp. 182 - 191, 2011.

[12] R. Mesleh, R. Mehmood, H. Elgala, and H. Haas, "Indoor MIMO Optical Wireless Communication Using Spatial Modulation," in IEEE International Conference on Communications (ICC), Cape Town, South Africa, May 22-27 2010, pp. 1-5.

[13] R. Mesleh, H. Elgala, and H. Haas, "Optical Spatial Modulation," IEEE/OSA J. Opt. Commun. Netw., vol. 3, no. 3, pp. 234-244, Mar. 2011, ISSN: 1943-0620.

[14] J. Jeganathan, A. Ghrayeb, L. Szczecinski, and A. Ceron, "Space Shift Keying Modulation for MIMO Channels," IEEE Trans. on Wireless Commun., vol. 8, no. 7, pp. 3692-3703, Jul. 2009.

[15] J. Jeganathan, A. Ghrayeb, and L. Szczecinski, "Generalized Space Shift Keying Modulation for MIMO Channels," in Proc. IEEE 19th Intern. Symp. on Personal, Indoor and Mobile Radio Commun. PIMRC 2008, Cannes, France, 15-18 Sep. 2008, pp. 1-5.

[16] T. Fath, M. Di Renzo, and H. Haas, "On the performance of space shift keying for optical wireless communications," in IEEE GLOBECOM Workshops (Globecom 2010) - Workshop on Optical Wireless Coтmunications, Miami, FL, USA, 6-10 Dec. 2010, pp. 990 - 994.

[17] W. Popoola, E. Poves, and H. Haas, "Generalised space shift keying for visible light communications," in 8th International Symposium on Communication Systems, Networks \& Digital Signal Processing (CSNDSP 2012), Poznan, Poland, 18-20 July 2012, pp. $1-4$.

[18] W. Popoola, E. Poves, and Haas, "Error Performance of Generalised Space Shift Keying for Indoor Visible Light Communications," IEEE Transactions on Communications, pp. 1-9, 2013.

[19] Y. Sun, D. Borah, and E. Curry, "Optimal symbol set selection in gssk visible light wireless communication systems," vol. 28 , no. 3, pp. 303306.

[20] K. Ebihara, K. Kamakura, and T. Yamazato, "Spatially-modulated spacetime coding in visible light communications using 22 led," in Circuits and Systems (APCCAS), 2014 IEEE Asia Pacific Conference on, Nov. 2014, pp. 320-323.

[21] W. Popoola and H. Haas, "Demonstration of the merit and limitation of generalised space shift keying for indoor visible light communications," vol. 32, pp. 1960-1965.

[22] S. Rajagopal, R. D. Roberts, and S.-K. Lim, "Ieee 802.15.7 visible light communication: Modulation schemes and dimming support," vol. 50, no. 3, pp. 72-82. 